\title{
Derivação vésico-amniótica no tratamento intra-uterino das uropatias obstrutivas: revisão e análise critica da experiência de um Centro de Medicina Fetal
}

\author{
Vesicoamniotic shunt in the intrauterine treatment of obstructive uropathies: \\ a review and critical analysis of the experience of the Fetal Medicine Center
}

\begin{abstract}
Alamanda Kfoury Pereira ${ }^{1}$, Gabriel Costa Ozanan ${ }^{2}$, Guilherme de Castro Rezende ${ }^{3}$,
\end{abstract} Zilma Silveira Nogueira Reis ${ }^{4}$, Henrique Vitor Leite ${ }^{5}$, Antônio Carlos Vieira Cabral ${ }^{6}$

\section{RESUMO}

Objetivo: avaliar a eficácia, a segurança e os benefícios do uso do cateter de derivação vésico-amniótico no tratamento intra-uterino das uropatias obstrutivas. Métodos: análise retrospectiva dos registros de 35 fetos portadores de uropatia obstrutiva, acompanhados em um centro de Medicina Fetal, no período compreendido entre 1990 e 2004, tratados pela insersão do cateter de derivação vésicoamniótica. As pacientes consentiram em submeter-se ao procedimento. Os casos selecionados seguiram os seguintes critérios de inclusão: gestação única; idade gestacional até 32 semanas; ausência de outras malformações; cariótipo normal; ultra-sonografia mostrando lesão obstrutiva no trato urinário, bilateral ou unilateral com comprometimento do rim contralateral, caracterizando hidronefrose (diâmetro ântero-posterior da pelve maior que $10 \mathrm{~mm}$ ), associada ou não a megaureter e megabexiga; oligoâmnio, dado por índice de líquido amniótico menor que 8; função renal normal, dada por critério ecográfico (aspecto dos rins à ecografia) e por estudo bioquímico da urina fetal (osmolaridade). Considerou-se normal a osmolaridade de até $210 \mathrm{mOsm}$ como indicativo de função renal preservada. Após o nascimento foram acompanhados pelo setor de Nefrologia Pediátrica do Hospital das Clínicas da UFMG. Os neomortos ou natimortos foram encaminhados para o setor de Anatomia Patológica. Foi realizada análise descritiva dos seguintes parâmetros: diagnóstico pré-natal da uropatia, idade gestacional à insersão do cateter, tempo de permanência do cateter, complicações pós-procedimento, mortalidade perinatal e sobrevida neonatal. Resultados: a válvula de uretra posterior foi a uropatia mais freqüente (62,8\%). A idade gestacional média da insersão do cateter foi 26,1 semanas. O tempo médio de permanência do cateter após a colocação até o parto foi de 46 dias (variando entre um e 119 dias). Ocorreram 4 mortes fetais e 17 mortes neonatais (mortalidade perinatal de $60 \%$ ). A principal causa dos óbitos foi a hipoplasia pulmonar. O oligoidrâmnio esteve presente em 33 dos 35 fetos acompanhados $(94,3 \%)$, tendo sido revertido em 23 casos $(70 \%)$, dos quais 14 sobreviveram ao período neonatal. Há 4 crianças em acompanhamento no setor de Nefrologia Pediátrica do Hospital das Clínicas da UFMG. Duas estão em diálise peritoneal, com expectativa de transplante renal, e duas apresentam-se com função renal preservada, com idades variando entre 2 meses e 4 anos. Conclusão: a derivação vésico-amniótica apresenta-se como uma alternativa viável de tratamento intra-uterino das uropatias graves, apresentando índice de sobrevida neonatal de $40 \%$ entre fetos que provavelmente evoluiriam para o óbito. Entretanto, o sucesso do procedimento esteve diretamente relacionado com a adequada seleção dos fetos e a precocidade da intervenção intrauterina, isto é, restringindo o procedimento àqueles fetos com menos de 32 semanas, com obstrução bilateral, sem malformações associadas e com função renal ainda preservada. A reversão do oligoidrâmnio não garantiu melhor prognóstico neonatal. Permanece controverso se a derivação vésico-amniótica é capaz de garantir a função renal preservada a longo prazo.

PALAVRAS-CHAVE: Uropatia fetal obstrutiva; Derivação vésico-amniótica; Feto/ anormalidades; Doenças fetais/ Obstrução uretral

\section{ABSTRACT}

Purpose: to analyze the efficacy, safety and real advantage of vesicoamniotic shunt catheter in the intrauterine treatment of obstructive uropathy. Methods: a retrospective and descriptive study, in which the evolution of 35 fetuses with obstructive

1 Professora Adjunta da Faculdade de Medicina da UFMG.

Médico Residente do Hospital das Clínicas da UFMG (MG)

Médico do Centro de Medicina Fetal do Hospital das Clinicas da UFMG (MG).

Médica do Hospital das Clínicas e do Centro de Medicina Fetal da UFMG (MG).

Professor Adjunto da Faculdade de Medicina da UFMG.

6 Professor Titular da Faculdade de Medicina da UFMG; Coordenador do Centro de Medicina Fetal da UFMG.

Correspondência: Alamanda Kfoury Pereira

Rua dos Otoni, 909, sala 2110 - Santa Efigênia - 30130-110 - Belo Horizonte - MG - e-mail: alamanda.k@terra.com.br

Recebido em: 23/4/2004

Aceito com modificações em: 4/4/2005 
uropathy, submitted to vesicoamniotic shunt from 1990 to 2004 in a Fetal Medical Center was evaluated. All these fetuses fitted the selection criteria defined by a protocol of this service, and had the parents' consent for the procedure. The Pediatric Nephrology Sector of the Hospital das Clínicas of UFMG assessed all of them after delivery to confirm the prenatal diagnosis and outcome. The dead neonates were studied by the Pathological Anatomy Sector of UFMG. Descriptive analysis of the following parameters was performed: prenatal diagnosis of the uropathy, gestational age at shunt insertion, time of catheter utilization, post-surgery complications, perinatal mortality and neonatal survival. Results: posterior urethral valve was the most common uropathy (62.8\%). The mean gestational age at the vesicoamniotic shunt placement was 26.1 weeks and the mean time of its presence was 46 days (1-119 days). There were four intrauterine fetal deaths and 17 in the neonatal period $(60 \%$ perinatal mortality). The main cause of death was pulmonary hypoplasia. Olygohidramnios was present in $33 / 35$ fetuses $(94.3 \%)$ and it was reversed in 23 of them (70\%); fourteen fetuses survived the neonatal period. At present, there are 4 children followed up by the Pediatric Sector of Nephrology of Hospital das Clínicas. Two of them have been treated with peritoneal dialysis, awaiting renal transplantation. The other two have normal renal function. Their age varies from 2 months to 4 years. Conclusion: the vesicoamniotic shunt may be a viable intrauterine treatment for severe obstructive uropathy, with $40 \%$ of survival rate of fetuses that might have progressed to death. However, the procedure's success was directly related to the adequate selection, and to the early intervention in the uterus, performed before 32 weeks of gestation in fetuses with bilateral obstruction, without any associated malformation and with still preserved renal function. Olygohidramnios reversion did not guarantee a good prognosis. It remains controversial if the vesicoamniotic shunt can really ensure long-term renal function.

KEYWORDS: Fetal obstructive uropathy; Fetal disiases; Urethral obstruction; Vesicoamniotic shunt; Intrauterine treatment; Fetus/abnormalities

\section{Introdução}

As uropatias obstrutivas compreendem heterogêneo grupo de anomalias de evolução e prognóstico variados, que afetam alguma porção do trato urinário. Incidem em $1 \%$ do total de gestações, das quais $20 \%$ tem significado clínico ${ }^{1}$. A obstrução crônica do trato urinário, dependendo do nível, extensão e duração, pode causar compressão progressiva do parênquima renal, limitando seu desenvolvimento e sua função, resultando em oligoidrâmnio, e hipoplasia pulmonar, com altas taxas de morbimortalidade perinatal ${ }^{1,2}$.

A ultra-sonografia é capaz de revelar não só detalhes anatômicos destas anomalias, como também sua evolução e aspectos de prognóstico, bem como métodos de tratamento intra-uterino, com o objetivo de preservar ou proteger a função renal até que o tratamento neonatal possa ser instituído, resultando em melhora significativa da evolução perinatal dos fetos comprometidos ${ }^{3-7}$.

Harrison et al. ${ }^{8,9}$ foram os primeiros a descrever experimentalmente os efeitos da obstrução do trato urinário sobre o desenvolvimento pulmonar e a demonstrar o efeito do alivio da obstrução intrauterina sobre a evolução destes fetos, principalmente quando realizado no periodo de nefrogênese ativa, isto é, entre 20 e 36 semanas de gestação. Outros estudos têm evidenciado que a intervenção pré-natal permite a sobrevida de fetos que iriam provavelmente ao óbito por hipoplasia pulmonar. Entretanto, permanece incerto se o tratamento intra-uterino pode interromper ou reverter alterações renais iniciadas antes deste período, de modo a não comprometer a função renal a longo prazo ${ }^{8-10}$.
A taxa de sucesso terapêutico é variável, devido a diferentes critérios metodológicos utilizados no diagnóstico e principalmente aos critérios de seleção dos fetos candidatos ao tratamento intra-uterino. Depende diretamente da precocidade diagnóstica, da gravidade do dano renal e do potencial de reversão destas lesões, apontando beneficio da intervenção naqueles fetos cuja função renal encontra-se preservada, porém ameaçada pela progressividade da doença ${ }^{3,4}$.

A taxa de complicações pós-procedimento está estimada em torno de $45 \% \%^{3,4,10}$, sendo as principais o deslocamento ou obstrução do cateter, rotura prematura das membranas, parto prematuro, morte fetal, gastrosquise iatrogênica, hemorragia, ascite urinária e lesões traumáticas ao feto ${ }^{6,9,11}$. O principal risco para a mãe é a infecção ${ }^{2}$, que pode eventualmente comprometer gestaçõs futuras. A questão que permanece controversa é se o tratamento intra-uterino realmente melhora a evolução da função renal pós-natal a longo prazo, embora sabendo que possa melhorar as chances de sobrevida perinatal. Não há estudos consistentes, randomizados ou multicêntricos, que avaliem a evolução a longo prazo dos fetos submetidos a este procedimento. Dependendo da idade gestacional, duração e gravidade da doença obstrutiva, o tratamento intra-uterino poderia estar apenas retardando um processo de insuficiência renal já instalado na vida intra-uterina, resultando em qualidade de vida pós-natal questionável.

O objetivo deste estudo foi analisar os efeitos do tratamento intra-uterino das uropatias obstrutivas, no Centro de Medicina Fetal do Hospital das Clínicas da UFMG nos últimos 14 anos. 


\section{Pacientes e Métodos}

Este estudo faz parte de linha de pesquisa do Centro de Medicina Fetal do Hospital das Clínicas da UFMG, aprovado pelo Comitê de Ética e Pesquisa da UFMG (COEP-UFMG) com o protocolo ETIC-232/02. De janeiro de 1990 até setembro de 2000, foram acompanhados, no Centro de Medicina Fetal da UFMG, 35 fetos portadores de uropatia obstrutiva, com indicações para a colocação intra-uterina do cateter de derivação. O diagnóstico ultra-sonográfico foi realizado em equipamento SONOACE 8800 (Medsom), pelo mesmo examinador, o qual também procedeu a estudo morfológico para avaliar o aspecto ecográfico dos rins e presença de anomalias associadas, e também à punção vesical sob visão ultra-sonográfica para estudo bioquímico da urina fetal. A cordocentese para cariótipo foi realizada para afastar cromossomopatia. As pacientes selecionadas foram previamente informadas sobre o diagnóstico da lesão e seu significado, a evolução natural da doença e prováveis seqüelas, bem como sobre o objetivo, segurança, resultados e possiveis riscos relacionados ao procedimento proposto. O tratamento foi realizado apenas após autorização da própria paciente e/ou familiar.

Os critérios de inclusão para o tratamento foram: gestação única, idade gestacional até 32 semanas; ausência de outras malformações e cariótipo normal.

Foram incluídos fetos com presença de lesão obstrutiva no trato urinário, bilateral ou unilateral com comprometimento do rim contralateral, manifesta pela presença de hidronefrose, associada ou não a megaureter ou megabexiga. Para o diagnóstico de hidronefrose utilizou-se o critério mais aceito, que considera a medida do diâmetro ântero-posterior de pelve renal de $10 \mathrm{~mm}$ ou mais, ou a presença de dilatação de cálices.

Para caracterização de oligoâmnio; foi utilizada a determinação do índice de líquido amniótico (ILA), ou seja, o somatório do diâmetro vertical dos maiores bolsões nos quatro quadrantes do abdome materno, considerando-se oligoidrâmnio se o valor deste somatório fosse menor que $8 \mathrm{~cm}$.

A função renal foi considerada normal, critério ecográfico (aspecto dos rins à ecografia) e por estudo bioquímico da urina fetal (osmolaridade), obtida por punção. A presença de microcistos na córtex renal e o aumento da ecogenicidade do parênquima renal comparado a estruturas vizinhas foi considerado sinal de displasia renal, significando, portanto, dano renal irreversível. A análise bioquímica da urina fetal foi feita por meio de punção simples com agulha da porção obstruída do trato urinário, guiada por ultra-sonografia, de acordo com a técnica descrita por Harrison et al. ${ }^{8}$. Para o estu- do bioquímico determinou-se a osmolaridade urinária, uma vez que reflete em conjunto a capacidade de eliminação e absorção urinária, realizada no laborarório do Centro de Medicina Fetal do HC-UFMG. A determinação da osmolaridade foi realizada em osmômetro digital, marca Mosmette Model 5004 (Precision Systems, Inc., Natick, MA, USA). As amostras foram analisadas colocando-se $5 \mathrm{~mL}$ do material em tubos próprios com pipeta automática, sendo necessários pelo menos 5 tubos por amostra. Após a calibração do aparelho com as soluções-padrão de 500 e 100 mOsm, as amostras foram medidas. O resultado foi dado pela média aritmética do resultado das 5 amostras. O erro do aparelho é de $2 \mathrm{mOsm} / \mathrm{kg}$. Considerou-se normal a osmolaridade de até $210 \mathrm{mOsm}$ como indicativa de função renal preservada ${ }^{8}$.

Os fetos que preencheram os critérios de inclusão foram submetidos à inserção do cateter de derivação desenvolvido no próprio serviço, cateter de Cabral e Leite, patenteado em 1992. Este consiste de cateter de polietileno, do tipo double pig-tail medindo $3 \mathrm{~cm}$ de comprimento e $3 \mathrm{~mm}$ de diametro, composto de duas espirais contendo duas hélices opostas em cada extremidade, separadas por seguimento retilineo. Este cateter é inserido dentro de agulha-guia, ecorrefringente a ultra-sonografia. Ao atingir o ponto obstruído, a agulha-guia vai sendo retirada enquanto o cateter vai sendo introduzido com a porção interna da agulha de punção.

As gestantes foram acompanhadas clinicamente, a intervalos de 7 a 14 dias até a resolução do parto. Foram analisadas as complicações gestacionais e os resultados perinatais, dados por óbito fetal, óbito neonatal, morte fetal depurada e sobrevida neonatal. Foram consideradas complicações pós-procedimento aquelas que ocorreram em intervalo de até uma semana após sua realização. Os recém-nascidos sobreviventes foram acompanhados no Serviço de Nefrologia Pediátrica do HC-UFMG, onde foram submetidos a propedêutica específica para identificar a lesão renal e as condições funcionais dos rins.

Com relação às características das pacientes incluídas no estudo, verifica-se que a idade materna variou de 15 a 38 anos, com média de 26, 6 anos. A paridade média foi de 3 filhos, variando de 1 a 7 .

\section{Resultados}

Foram 35 fetos submetidos ao tratamento intra-uterino da uropatia obstrutiva. A idade gestacional média de diagnóstico foi 25 semanas (Tabela 1) e a válvula de uretra posterior, descrita como obstrução uretral, a uropatia mais freqüen- 
te, encontrada em $62,8 \%$ dos casos (Tabela 2). A idade gestacional média de inserção do cateter foi de 26,1 semanas, com variação entre 18 e 33 semanas de gestação. O tempo médio de permanência do cateter após a colocação até o parto foi de 46 dias (variando entre um e 119 dias). Ocorreram 16 complicações gestacionais decorrentes do procedimento, relacionados na Tabela 3 .

Tabela 1 - Distribuição das pacientes de acordo com a idade gestacional ao diagnóstico da lesão fetal.

\begin{tabular}{crr}
\hline $\begin{array}{c}\text { Idade gestacional ao } \\
\text { diagnóstico (semanas) }\end{array}$ & Número & $\%$ \\
\hline$<17$ & 3 & 8,6 \\
$17-24$ & 11 & 31,4 \\
$24-30$ & 15 & 42,9 \\
$\geq 30$ & 5 & 14,1 \\
Total & 35 & 100 \\
\hline
\end{tabular}

Tabela 2 - Distribuição das pacientes de acordo com o diagnóstico morfológico prénatal da uropatia obstrutiva.

\begin{tabular}{lcr}
\hline Uropatias obstrutivas & Número & $\%$ \\
\hline OJUP & 3 & 8.6 \\
OJUV & 10 & 23.6 \\
Obstrução Uretral & 22 & 62.8 \\
Total & 35 & 100 \\
\hline
\end{tabular}

OJUP- obstrução da junção uretero-pélvica; OJUV- obstrução da junção uretero-vesical.

Tabela 3 - Complicações gestacionais decorrentes da insersão do catéter de derivação vésico-amniótico.

\begin{tabular}{lcc}
\hline Complicação & Número & $\%$ \\
\hline Descolamento prematuro de membranas & 1 & 2,9 \\
Rotura prematura de membranas & 1 & 2,9 \\
Trabalho de parto prematuro & 3 & 8,5 \\
Ascite fetal & 1 & 2,9 \\
Fibrose do parênquima renal & 1 & 2,9 \\
Reacúmulo de líquido no trato urinário & 7 & 20 \\
Mal posicionamento/migração do catéter & 2 & 5,7 \\
Sem complicações & 19 & 54,3 \\
Total & 16 & 45,7 \\
\hline
\end{tabular}

Houve quatro casos de morte intra-uterina $(11,4 \%)$, dos quais dois tiveram o diagnóstico anatomopatológico confirmando o diagnóstico de válvula de uretra posterior, porém não se conseguiu determinar a provável causa mortis. Um feto apresentava, além da válvula de uretra posterior, genitália ambigua e ascite urinosa. A morte neonatal ocorreu em 17 recém-nascidos $(48,6 \%)$, dos quais sete apresentavam outras malformações associadas, que não haviam sido detectadas no pré-natal. A hipoplasia pulmonar foi a responsável direta pelo óbito neonatal em 15 recém-nascidos (88\%). Dos 15 casos nos quais a hipoplasia pulmonar esteve pre- sente, nove apresentaram restauração do volume do líquido amniótico após a colocação do cateter, um apresentava volume de liquido normal e em três casos não houve restauração do mesmo.

Das 35 gestações estudadas, o oligoidrâmnio esteve presente em 33 (94,3\%). O líquido amniótico foi restaurado em 23 casos (70\%). Porém, destes 23 casos, nove foram a óbito por hipoplasia pulmonar, mesmo tendo sido restaurado o volume de líquido amniótico (Tabela 4).

Tabela 4 - Resultado perinatal da insersão do catéter de derivação vésico-amniótica.

\begin{tabular}{lcc}
\hline Complicação & Número & $\%$ \\
\hline Morte fetal & 4 & 11,4 \\
Morte neonatal & 17 & 48,6 \\
${ }^{*}$ Morte neonatal depurada & 10 & 28,6 \\
Sobrevida neonatal & 14 & 40 \\
Total & 35 & 100 \\
\hline${ }^{*}$ Excluídas as mortes neonatais associadas a outras anomalias.
\end{tabular}

Quatorze fetos sobreviveram no período neonatal $(40 \%)$. Foram acompanhados durante o primeiro mês de vida, não apresentando, durante esse periodo, qualquer alteração renal ou pulmonar atribuída à lesão obstrutiva ou ao procedimento intrauterino. Dos 14 neonatos que sobreviveram, 5 $(35,7 \%)$ apresentavam obstrução da junção ureteropélvica, oito $(57,2 \%)$ eram portadores de válvula de uretra posterior e um $(7,1 \%)$ apresentava obstrução da junção uretero-vesical. Houve perda do seguimento de dez neonatos a partir do primeiro mês de vida. Atualmente, quatro crianças estão sendo acompanhadas no setor de Nefrologia Pediátrica do Hospital das Clínicas da UFMG.

Uma delas está com 2 anos e 6 meses, com insuficiência renal crônica, em diálise peritoneal. Durante o pré-natal, foi encaminhada na $30^{\mathrm{a}}$ semana de gestação com hidronefrose bilateral acentuada, acompanhada de megaureter e megabexiga, oligoâmnio e rim esquerdo de aspecto ecográfico displásico. A osmolaridade urinária foi de 209 mOsm. Implantou-se o cateter de derivação na $31^{a}$ semana, com reversão do oligoâmnio, porém sem reversão dos outros parâmetros. Após o nascimento, o diagnóstico de válvula de uretra posterior foi confirmado, com função renal comprometida. Foi realizada de imediato ureterostomia bilateral e no $25^{\circ}$ dia de vida foi realizada nefrectomia à esquerda. Meses depois foi corrigida a válvula de uretra posterior e o fechamento das ureterostomias. Atualmente a criança apresenta-se com escórias nitrogenadas e ionograma controlados, em diálise peritoneal. Possui déficit nutricional e de crescimento moderados. As perspectivas são de manter o quadro estável até que a criança alcance idade para submeter-se a transplante renal com sucesso. 
Outra criança está com 3 anos, em diálise peritoneal, também à espera do momento adequado para realização de transplante renal. Esta criança durante o pré-natal apresentou hidronefrose bilateral, megaureteres e megabexiga, oligoâmnio e rins displásicos a ultra-sonografia, porém osmolaridade de $201 \mathrm{mOsm}$. A colocação do cateter ocorreu com 28 semanas de gestação, porém não houve a reversão esperada do oligoâmnio. Após o nascimento, detectou-se função renal comprometida, mas ainda residual, e diagnóstico confirmado de válvula de uretra poste-rior, que foi corrigida após o nascimento.

Outras duas crianças estão em acompanhamento clínico, com função renal preservada, uma com 2 meses e a outra com 4 anos de idade.

\section{Discussão}

As uropatias obstrutivas têm despertado grande interesse em medicina fetal, seja pela freqüência com que são identificadas no pré-natal, seja pelo leque de opções terapêuticas de que se dispõe. A ultrasonografia é capaz de estabelecer o diagnóstico com segurança e eficácia, podendo determinar a natureza, o nível e a intensidade da lesão obstrutiva. Permite também avaliar a função renal, seja pela imagem ecográfica dos rins e pelo volume de líquido amniótico, seja auxiliando a punção para coleta da urina fetal para estudo bioquímico. Entre as opções terapêuticas estão o acompanhamento seriado, a antecipação do parto e o tratamento cirúrgico intra-uterino. Este consiste na punção da porção obstruída e a insersão de cateter de derivação (shunt) entre esta e a cavidade amniótica, sob visão ultra-sonográfica, permitindo a descompressão do parênquima renal até que o tratamento definitivo seja realizado após o nascimento. Os objetivos principais do shunt são evitar a displasia renal e a hipoplasia pulmonar, que são as principais causas de morte destes fetos ${ }^{3,4,8}$.

O sucesso da cirurgia intra-uterina passa diretamente pela seleção adequada dos fetos para o procedimento. Certamente os resultados são melhores quando ainda não houve degradação renal expressi$\mathrm{va}^{3,11}$. Não há uniformidade entre os autores quanto aos critérios de seleção dos fetos para o tratamento, porém os utilizados neste estudo são consensuais. Deste modo, indicam-se para tratamento intrauterino fetos prematuros (menos de 32 semanas), sem malformações associadas, apresentando lesões graves o suficiente (bilaterais, com oligoidrâmnio), porém que não tenham tido perda da função renal. Utilizamos critérios ultra-sonográficos (lesão bilateral, menos de 32 semanas, ausência de malformações associadas, aspecto ecográfico normal dos rins e volume de líquido amniótico diminuído) e a medida da osmolaridade (até $210 \mathrm{mOsm} / \mathrm{ml}$ ). Embora estu- dos demonstrem alta sensibilidade da dosagem de cálcio e maior especificidade do sódio em predizer a displasia renal pós-natal, utilizamos a osmolaridade, por refletir em conjunto a função tubular e glomerular do rim, portanto apresenta boa acuidade global em predizer a função renal. Walsh e Johnson ${ }^{5}$ acrescentam como critério de seleção fetos do sexo masculino ${ }^{5}$.

Observa-se neste estudo que 20 dos 35 fetos selecionados para tratamento apresentam idade gestacional de mais de 24 semanas, o que reflete referenciamento tardio ao serviço, com o risco de que o tratamento pode não ser tardio para garantir a sobrevida neonatal, mas pode ser tardio para garantir sobrevida a longo prazo, com boa função renal. A doença mais prevalente é a obstrução uretral de válvula de uretra posterior, concordante com outras séries ${ }^{12}$.

Houve neste estudo 16 (45,7\%) casos que apresentaram complicações gestacionais, concordante com outras séries ${ }^{2,4,8,10,11}$, sendo as mais freqüentes o trabalho de parto prematuro e a migração do cateter. Parece que as dificuldades técnicas decorrentes do oligoidrâmnio e do posicionamento do feto podem ter contribuído para a ocorrência destas complicações.

Em relação aos resultados perinatais, ocorreram neste estudo quatro mortes intra-uterinas $(11,4 \%)$, das quais 2 tiveram confirmado o diagnóstico de válvula de uretra posterior, um deles com ascite urinosa e genitália ambígua, porém não se conseguiu determinar com segurança qual foi a causa do óbito. É possivel que, mesmo apresentando os critérios para o tratamento, estes fetos já se encontrassem numa fase em que mesmo com a colocação do cateter o processo de insuficiência renal/pulmonar já estaria desencadeado. É este o motivo pelo qual a idade gestacional limite de 32 semanas tem sido considerada, por alguns auto$\mathrm{res}^{4}$, tardia para indicar o procedimento. Outros estudos demonstram casos de falha em reverter a insuficiência renal após a colocação do cateter ${ }^{8,9,10}$.

A morte neonatal ocorreu em 17 recém-nascidos $(48,6 \%)$, dos quais quase a metade apresentava outras malformações associadas. Portanto, a morte neonatal depurada foi de quase 30\% (10 casos). Os trabalhos pioneiros de Harrison et al. ${ }^{8,9}$ demonstraram que os fetos tratados, que teriam sobrevida de $0 \%$, apresentaram sobrevida perinatal de $40 \%$. Coplen ${ }^{4}$, em revisão de 5 grandes estudos, verificou taxa global de sobrevida pós-shunt de $47 \% \%^{2}$, além de risco de complicações em $45 \%$ dos casos. O Sistema de Registro Internacional de Cirurgia Fetal descreveu 73 fetos portadores de uropatias obstrutivas graves, tratados com a colocação de shunt vésico-amniótico. O índice de sobrevida perinatal foi de $41 \%$, podendo chegar a $70 \%$ com a seleção adequada dos fetos ${ }^{11}$. Estudo realizado em 1997 neste 
serviço apresentou resultado semelhante.

O tratamento intra-uterino foi realizado em 25 fetos portadores de uropatia obstrutiva, com sobrevida perinatal de $40 \%$ e incidência de complicações pósprocedimento em $48 \%$ dos casos. A falha em restaurar o oligoidrâmnio associou-se a 100\% de mortalidade ${ }^{13}$. Clark et al. ${ }^{12}$, em artigo de revisão, encontraram 16 estudos observacionais (nove séries e sete estudos controlados), totalizando 342 fetos tratados. Os estudos controlados compararam em tabela 2 X 2 a evolução perinatal dos fetos tratados com relação aos não tratados. Demonstraram que a drenagem intra-uterina, por meio da colocação do shunt, melhorou os índices de sobrevida perinatal.

Os estudos de séries mostraram significativa melhora na sobrevida perinatal mesmo do subgrupo de fetos de prognóstico ruim, decorrente da preservação da função pulmonar ${ }^{12}$. Neste estudo, a hipoplasia pulmonar foi a principal causa da morte em 15 dos 17 neonatos (88\%), dos quais nove apresentaram restauração do volume do líquido amniótico após a colocação do cateter, um apresentava volume de líquido normal e em três casos não houve restauração do mesmo. Parece que há outros fatores envolvidos entre a obstrução do trato urinário e a hipoplasia pulmonar, além do oligoidrâmnio, demonstrando que esta grave complicação nem sempre é revertida com a restauração do volume de líquido amniótico.

Verificamos significativa perda de seguimento das crianças que sobreviveram ao período neonatal, o que limita nossa discussão quanto à validade do tratamento a longo prazo. Porém, é possivel verificar que temos um referenciamento tardio, e mesmo com critérios definidos de seleção para tratamento, há casos em que este é tardio. Por outro lado, estas crianças que se apresentam em diálise peritoneal e candidatas a transplante renal teriam certamente evoluído para o óbito se não tivessem sido tratadas no pré-natal.

Não estão definidas as vantagens a longo prazo da intervenção intra-uterina ${ }^{14}$. Outros estudos prospectivos, que acompanhem estes fetos por vários anos, se fazem necessários, para que se defina com maior clareza se a colocação do cateter intra-útero de fato seria eficaz para preservar a função renal futura do concepto.

A derivação vésico-amniótica apresenta-se como alternativa viável de tratamento intra-uterino das uropatias graves, com indice de sobrevida neonatal de $40 \%$ de fetos que provavelmente evoluiriam para o óbito. Entretanto, o sucesso do procedimento esteve diretamente relacionado com a adequada seleção dos fetos e a precocidade da intervenção intra-uterina, isto é, restringindo o procedimento àqueles fetos abaixo de 32 semanas, com obstrução bilateral, sem malformações associadas e com função renal ainda preservada. A reversão do oligoâmnio não garantiu melhor prognóstico neonatal. Permanece controverso se a derivação vésico-amniótica é capaz de garantir a função renal preservada a longo prazo.

\section{Referências}

1. Gonçalves LF, Jeanty P, Piper JM. The accuracy of prenatal ultrasonography in detecting congenital anomalies. Am J Obstet Gynecol. 1994;171(6):1606-12.

2. Mouriquand PD, Troisfontaines E, Wilcox DT. Antenatal and perinatal uro-nephrology: current questions and dilemmas. Pediatr Nephrol. 1999;13(9):938-44.

3. Shokeir AA, Nijman RJ. Antenatal hydronephrosis: changing concepts in diagnosis and subsequent management. BJU Int. 2000;85(8):987-94.

4. Coplen DE. Prenatal intervention for hydronephrosis. J Urol. 1997;157(6):2270-7.

5. Walsh DS, Johnson MP. Fetal intervention for obstructive uropathy. Semin Perinatol. 1999;23(6):484-95.

6. Lewis KM, Pinckert TL, Cain MP, Ghidini A. Complications of intrauterine placement of a vesicoamniotic shunt. Obstet Gynecol. 1998;91(5 Part 2):825-7.

7. Irwin BH, Vane DW. Complications of intrauterine intervention for treatment of fetal obstructive uropathy. Urology. 2000;55(5):774-6.

8. Harrison MR, Nakayama DK, Noall RA, de Lorimier AA. Correction of congenital hydronephrosis in utero. II. Decompression reverses the effects of obstruction on the fetal lung and urinary tract. J Pediatr Surg. 1982;17:965-74.

9. Harrison MR, Filly RA. The fetus with obstructive uropathy: pathophysiology, natural history, selection and treatment. In: Harrison MR, Golbus MS, Filly RA, editors. The unborn patient: prenatal diagnosis and treatment. $2^{a}$ ed. Philadelphia: Saunders; 1994. p. 328-61.

10. Makino Y, Kobayashi H, Kyono K, Oshima K, Kawarabayashi T. Clinical results of fetal obstructive uropathy treated by vesicoamniotic shunting. Urology. 2000;55(1):118-22.

11.Manning FA, Harrison MR, Rodeck C. Catheter shunts for fetal hydronephrosis and hydrocephalus. Report of International Fetal Surgery Registry. N Engl J Med. 1986;315(5):336-40.

12. Clark TJ, Martin WL, Divakaran TG, Whittle MJ, Kilby MD, Khan KS. Prenatal bladder drainage in the management of fetal lower urinary tract obstruction: a systematic review and meta-analysis. Obstet Gynecol. 2003;102(2):367-82.

13.Lopes APBM. Tratamento dos uropatias obstrutivas fetais utilizando o cateter desenvolvido no centro de medicina fetal Hospital das Clínicas da UFMG [dissertação]. Belo Horizonte: Universidade Federal de Minas Gerais; 1997.

14.Freedman AL, Johnson MP, Smith CA. Long-term outcome in children after antenatal intervention for obstructive uropathies. Lancet. 1999;354(9176):374-7. 